\title{
Avaliação da completitude das variáveis da declaração de óbitos de menores de um ano residentes em Pernambuco, 1997-2005
}

\author{
Completeness variable evaluation from death certificate \\ of children less than one year in Pernambuco State, 1997-2005
}

Juliana M artins Barbosa da Silva Costa ${ }^{1}$

Paulo Germano deFrias ${ }^{1}$

${ }^{1}$ Instituto M aterno-Infantil Professor Fernando

Figueira. Rua dos Coelhos 300, Boa Vista. 50070-550 Recife PE.

jumarbs@ibest.com.br
Abstract An evaluative, descriptive, time series study was conducted to evaluate the completeness of variables from death certificate. The study population consisted of all deaths of children less than one year old living in Pernambuco State, Brazil, from 1997 to 2005, contained in the database of the M ortality Information System (SIM ), provided by the Data Processing D epartment of theU nified Health System (DATASUS). We calculated each variable's incompleteness (blank/unknown data) by municipality of residence. Based on these data, we calculated the percentage of municipalities with excellent completeness (>95\%) and the tendency qui-square ( $p>95 \%)$. There was a difference in the filling behavior of the variables of the death certificate. While sex, age, race/color, place of occurrence, type of delivery and weeks of gestation had an increase in the percentage of municipalities with excellent filling, death confirmed by autopsy, completion of surgery, complement and medical examination showed a decrease. The study emphasizes the importance of educational actions and a better control in the filling of the variables in death certificates.

Key words Evaluation, Information systems, Vital statistics
Resumo Com o objetivo deavaliar a completitude das variáveis da declaração de óbito de menores de um ano residentes em Pernambuco, foi realizado um estudo avaliativo, descritivo de série temporal. A população do estudo foi composta pelo total de óbitos de menores de um ano residentes em Pernambuco, no período de 1997 a 2005, contidos na base de dados do Sistema de Informações sobre M ortalidade, disponibilizados pelo departamento de informática do SU S (DATASUS). Foi calculada a incompletude (branco/ignorado) de cada variável segundo município de residência. Com base nesses dados, foi calculado o percentual de municípios com preenchimento excelente ( $>95 \%$ ) e o qui-quadrado detendência ( $p>95 \%$ ). O bservou-se um comportamento difer enciado no preenchimento das variáveis da declaração de óbito. Enquanto as variáveis sexo, idade, raça/ cor, local de ocorrência, tipo de parto e semanas de gestação apresentaram um incremento no percentual de municípios com preenchimento excelente, as variáveis óbito confirmado por necropsia, realização de cirurgia, exame complementar e assistência médica apresentaram um decréscimo. Ressalta-sea necessidade da adoção de medidas educativas e um maior controle no preenchimento da declaração de óbito.

Palavras-chave Avaliação, Sistemas de informação, Estatísticas vitais 


\section{Introdução}

As estatísticas de mortalidade são uma importante ferramenta para o planejamento e avaliação das ações e serviços de saúde. Historicamente, as informações sobre mortalidade são coletadas, analisadas e divulgadas pelo I nstituto Brasileiro de Geografia eEstatística (IBGE). Contudo, devido às limitações dos dados disponibilizados quenão permitiam a descrição detalhada do perfil epidemiológico do país, o M inistério da Saúde criou, em 1975, o Sistema de Informação sobre M ortalidade (SIM), com um modelo padronizado de declaração de óbito ${ }^{1}$.

A declaração de óbito éo instrumento oficial de coleta de dados do SI M. O fluxo de encaminhamento dessas declarações, bem como as normas quanto ao seu preenchimento e o processamento das informações, são definidos pelo $\mathrm{M} \mathrm{i-}$ nistério da Saúde e estão contemplados nos manuais de procedimentos ${ }^{2}$ e de preenchimento ${ }^{3}$ da declaração de óbito.

A implantação do SIM permitiu a captação, em âmbito nacional, de informações contínuas sobre as características sociais, demográficas e epidemiológicas dos óbitos infantis ${ }^{1,4}$, que possibilita o monitoramento, um maior detal hamento e compreensão da mortalidade infantil e seus determinantes.

Essa variedade de informações subsidia a construção dos principais indicadores utilizados para a análise da situação de saúde infantil. Entre estes, destacam-se a mortalidade proporcional por causas e o coeficiente de mortalidade infantil. Entretanto, o sistema necessita de uma boa cobertura, regularidade e de que as declarações de óbito estejam com todos os campos preenchidos de forma correta para que as conclusões retiradas de sua análise se aproximem da realidade local ${ }^{4}$.

Alguns estudos têm demonstrado fragilidades no SIM, o que limita sua utilização para o cálculo de indicadores confiáveis. Os problemas citados com mai or frequência se referem à baixa cobertura ${ }^{5,6}$, com destaque para o subrregistro em menores de um ano ${ }^{7,8}$; fal has na declaração da causa básica e o elevado percentual de causas mal definidas ${ }^{6,9} ;$ erros de preenchimento da declaração de óbito ${ }^{1}$ e a elevada incompletude das variáveis ${ }^{9-13}$. Esses problemas não são homogêneos no território nacional. As regiões N orte e N ordeste apresentam maiores dificuldades na operacionalização do sistema, fato também constatado entre municípios de diferentes portes populacionais situados em uma mesma macrorre- gião e entre áreas urbanas e rurais. Essa situação evidencia as desi gual dades e os diferentes estágios de desenvolvimento que se encontram em várias localidades do país ${ }^{12,14 \cdot 16}$.

Diante do exposto, o presente estudo pretende avaliar a completitude no preenchimento das variáveis da declaração de óbito de menores de um ano residentes em Pernambuco no período de1997 a 2005, por meio da identificação do quantitativo de municípios que apresentam um grau depreenchimento excelentee como esteestá evoluindo. Busca-se, dessa forma, subsidiar análises mais fidedignas, além de estimular o correto preenchimento da declaração de óbito, melhorando as informações do SIM .

\section{Métodos}

Foi realizado um estudo avaliativo, descritivo de série temporal. A população de estudo foi composta pelo total de registros de óbitos de menores de um ano residentes em Pernambuco, no período de 1997 a 2005, contidos na base de dados do SIM disponibilizados pelo Ministério da Saúde, por meio da página do DATASU ${ }^{17}$.

0 ano de 1997 foi escolhido por ser 0 ano que marca a mais recente conformação político-administrativa de Pernambuco. Até 1995, existiam no estado 177 municípios. N esteano, foram criados oitos municípios por meio da Lei Estadual Complementar $n^{0} 1^{18}$. Contudo, a implantação das sedes municipais ocorreu apenas em janeiro de 1997, passando Pernambuco a ser composto por 185 municípios, atualmente distribuídos em onze Gerências Regionais de Saúde (GERES). 0 ano de 2005 foi o último ano com dados disponíveis para análise.

A partir das informações contidas no SIM, foi construído um banco de dados com variáveis relacionadas à criança (sexo, idade, raça/cor e peso ao nascer), a al guns atributos maternos (idade materna e número de filhos tidos - nascidos vivos e nascidos mortos), à gestação e ao parto (semanas de gestação e tipo de parto) e ao momento do óbito (local de ocorrência, assistência médica, óbito confirmado por exame complementar, óbito confirmado por realização de cirurgia, óbito confirmado por necropsia). Osconceitos e definições adotados em cada variável foram os preconizados pelo M inistério da Saúde, contidos no manual de preenchimento do $\mathrm{SI} \mathrm{M}^{3}$.

Cada variável foi tabulada, tendo-se calculado o percentual deincompletude (preenchimento em branco/ignorado) por município de resi- 
dência. Valendo-se dessas informações, foi calculado o percentual de municípios que possuíam um grau de preenchimento considerado excelente ( $>95 \%$ ), tendo como base o sistema de escores proposto por Romero e Cunha ${ }^{12}$. Esses autores adotaram como ponto de referência para avaliar a incompletude os seguintes escores: excelente, quando a variável possuía menos de $5 \%$ de preenchimento incompleto, bom (5 a 10\%), regular ( 10 a $20 \%$ ), ruim ( 20 a $50 \%$ ) e muito ruim (mais 50\%). Foram excluídos os municípios nos quais ocorreram menos de seis óbitos/ ano devido à influência dos pequenos números no cálculo dos escores.

Com essas informações, foram construídas as séries temporais de cada variável. Para análise estatística, foi calculado o qui-quadrado de tendência, com nível designificância de $5 \%$, quepermite identificar se o aumento ou decréscimo observado ocorreu de forma homogênea ao longo do tempo.

0 referido estudo foi submetido e aprovado pelo Comitê de Ética e Pesquisa do Instituto Medicina Integral de Pernambuco Profo Fernando Figueira(IMIP).

\section{Resultados}

No período de 1997 a 2005, o preenchimento das variáveis da declaração de óbito não apresentou um padrão bem definido. Algumas variáveis, como as relacionadas à criança, à gestação e ao parto, apresentaram um incremento no número de municípios com preenchimento excelente (>95\%). Por outro lado, grande parte das variáveis relacionadas a alguns atributos maternos mantiveram-se estáveis e as relacionadas ao momento do óbito apresentaram uma diminuição no número demunicípios com preenchimento excelente (Tabelas 1, 2 e 3).

Entre as variáveis relacionadas à criança, 0 sexo e a idade do recém-nascido apresentaram os maiores percentuais de municípios com pre enchimento excelente. Apesar do qui-quadrado detendência não ter sido estatisticamentesignificante, devido a oscilações ocorridas no período, no ano de 2005, 96,5\% dos municípios apresentaram um preenchimento excelente da variável sexo e 100,0\% dos municípios da variável idade. As variáveis raça/cor e peso ao nascer apresentaram um incremento importante no percentual demunicípios com preenchimento excelente, passando de $2,0 \%$ e 3,3\% em 1997 para 33,6\% e $13,3 \%$ em 2005 ( $\chi^{2}=171,13$ e 50,59 $\left.p<0,005\right)$, respectivamente. Apesar do incremento observado, ainda são poucos os municípios que preenchem estas variáveis de maneira satisfatória (Tabela 1).

De uma forma geral, as variáveis relacionadas a alguns atributos maternos apresentaram 0 menor número demunicípios com prenchimen-

Tabela 1. Percentual de municípios com preenchimento excelente ( $>95 \%$ ) e qui-quadrado de tendência segundo variáveis relacionadas à criança da declaração de óbito. Pernambuco, 1997 a 2005.

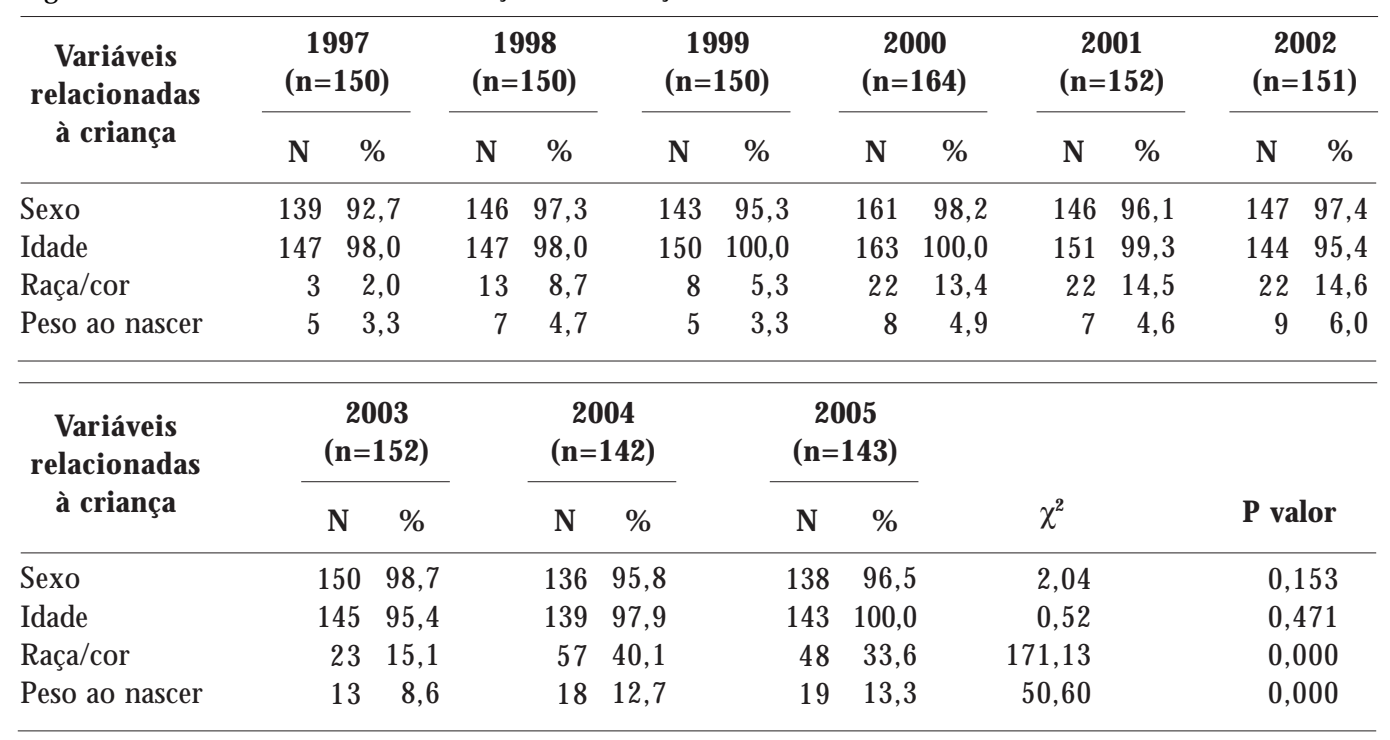

\footnotetext{
* Foram excluídos os municípios em que ocorreram < 6 óbitos/ano.
} 
to excelente. Apenas a escolaridadematerna apre sentou um incremento no número de municípi- os com preenchimento excelente, passando de nenhum município, em 1997, para 23,1\%, em

Tabela 2. Percentual de municípios com preenchimento excelente (>95\%) e qui-quadrado de tendência segundo variáveis relacionadas a alguns atributos maternos da declaração de óbito. Pernambuco, 1997 a 2005.

\begin{tabular}{|c|c|c|c|c|c|c|c|c|c|c|c|c|}
\hline \multirow{2}{*}{$\begin{array}{l}\text { Variáveis } \\
\text { relacionadas a al guns } \\
\text { atributos maternos }\end{array}$} & \multicolumn{2}{|c|}{$\begin{array}{c}1997 \\
(n=150)\end{array}$} & \multicolumn{2}{|c|}{$\begin{array}{c}1998 \\
(n=150)\end{array}$} & \multicolumn{2}{|c|}{$\begin{array}{c}1999 \\
(n=150)\end{array}$} & \multicolumn{2}{|c|}{$\begin{array}{c}2000 \\
(n=164)\end{array}$} & \multicolumn{2}{|c|}{$\begin{array}{c}2001 \\
(n=152)\end{array}$} & \multicolumn{2}{|c|}{$\begin{array}{c}2002 \\
(n=151)\end{array}$} \\
\hline & $\mathrm{N}$ & $\%$ & $\mathrm{~N}$ & $\%$ & $\mathrm{~N}$ & $\%$ & $\mathrm{~N}$ & $\%$ & $\mathrm{~N}$ & $\%$ & $\mathrm{~N}$ & $\%$ \\
\hline Idade materna & 16 & 10,7 & 19 & 12,7 & 13 & 8,7 & 19 & 11,6 & 15 & 9,9 & 21 & 13,9 \\
\hline $\begin{array}{l}\text { Escolaridade materna } \\
\text { Filhos tidos }\end{array}$ & - & - & 1 & 0,7 & 6 & 4,0 & g & 5,5 & 5 & 3,3 & 8 & 5,3 \\
\hline Nascido vivo & 1 & 0,7 & 4 & 2,7 & 2 & 1,3 & 4 & 2,4 & - & - & 1 & 0,7 \\
\hline Nascido morto & - & - & - & - & - & - & & - & - & - & - & - \\
\hline \multirow{2}{*}{$\begin{array}{l}\text { Variáveis } \\
\text { relacionadas a al guns } \\
\text { atributos maternos }\end{array}$} & \multicolumn{3}{|c|}{$\begin{array}{c}2003 \\
(n=152)\end{array}$} & \multicolumn{2}{|c|}{$\begin{array}{c}2004 \\
(n=142)\end{array}$} & \multicolumn{3}{|c|}{$\begin{array}{c}2005 \\
(n=143)\end{array}$} & \multirow{2}{*}{\multicolumn{2}{|c|}{$\chi^{2}$}} & \multirow{2}{*}{\multicolumn{2}{|c|}{ P valor }} \\
\hline & & $\mathrm{N}$ & $\%$ & $\mathrm{~N}$ & $\%$ & & $\mathrm{~N}$ & $\%$ & & & & \\
\hline Idade materna & & 15 & 9,9 & 22 & 15,5 & & 15 & 10,5 & \multicolumn{2}{|c|}{0,053} & \multicolumn{2}{|c|}{0,817} \\
\hline $\begin{array}{l}\text { Escolaridade materna } \\
\text { Filhos tidos }\end{array}$ & & 12 & 7,9 & 10 & 7,0 & & 15 & 10,5 & \multicolumn{2}{|c|}{14,580} & \multicolumn{2}{|c|}{0,000} \\
\hline Nascido vivo & & - & - & 1 & 0,7 & & 5 & 3,5 & \multicolumn{2}{|c|}{0,892} & \multicolumn{2}{|c|}{0,345} \\
\hline Nascido morto & & - & - & - & - & & - & - & & $*$ & \multicolumn{2}{|c|}{$*$} \\
\hline
\end{tabular}

*Foram excluídos os municípios em queocorreram < 6 óbitos/ano.

Tabela 3. Percentual de municípios com preenchimento excelente ( $>95 \%$ ) e qui-quadrado de tendência segundo variáveis relacionadas a gestação e ao parto da declaração de óbito. Pernambuco, 1997 a 2005.

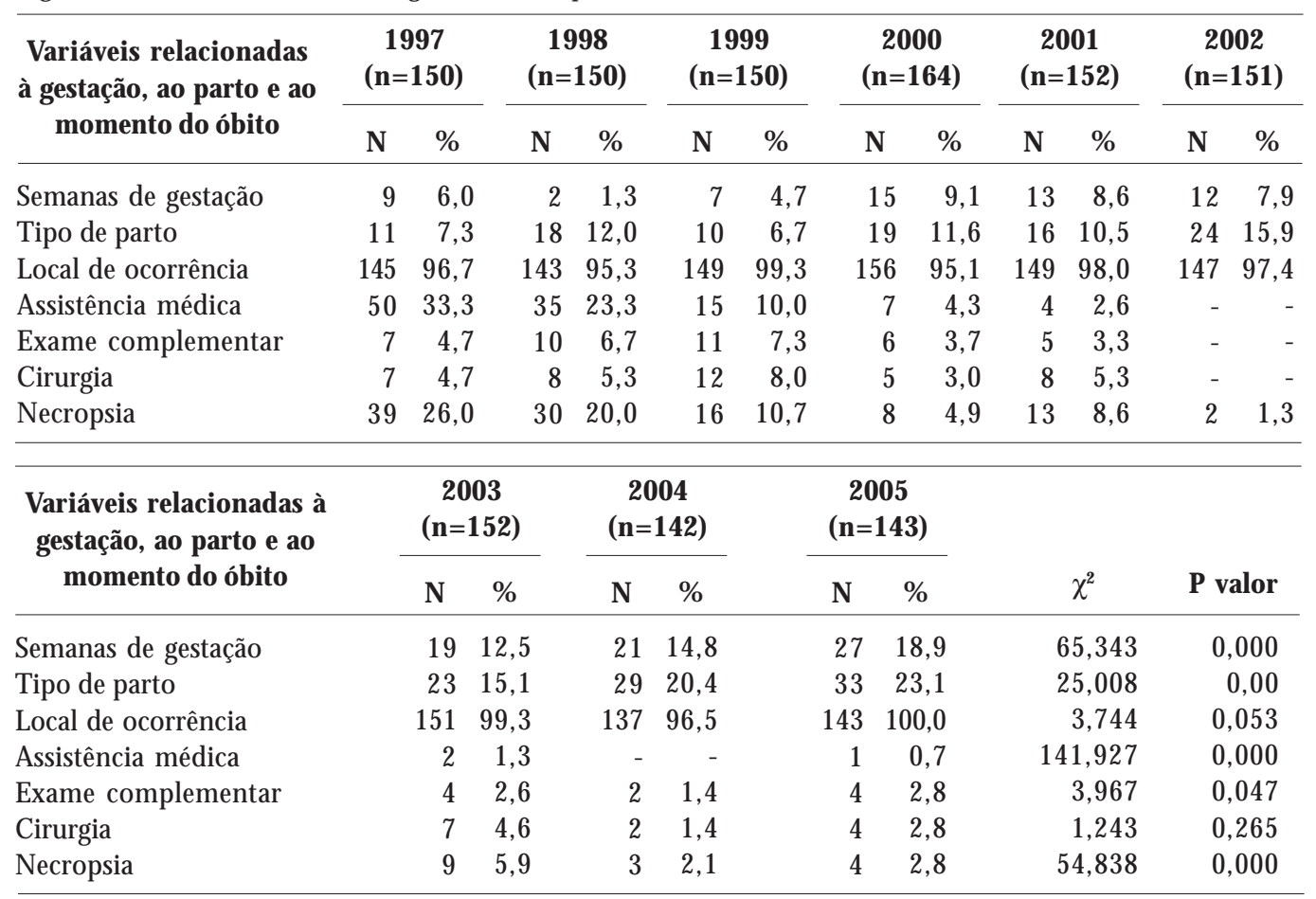

*Foram excluídos os municípios em queocorreram < 6 óbitos/ano. 
$2005\left(\chi^{2}=14,580 p<0,0001\right)$. A idade materna não apresentou significância estatística, mantendo um percentual de aproximadamente $10,5 \%$ dos municípios com preenchimento excelenteao longo do período analisado. Destaca-se ainda a variável número de filhos tidos, que em ambos os subcomponentes, número de filhos nascidos vivos e número de filhos nascidos mortos, apresentou os mais baixos percentuais demunicípios com preenchimento excelente entre todas as variáveis estudadas (Tabela 2).

As variáveis relacionadas à gestação e ao parto apresentaram um aumento no número de municípios com preenchimento excelente; entretanto, estes ainda são em número reduzido. 0 tipo de parto passou de $7,3 \%$ dos municípios, em 1997, para 23,1\%, em 2005 ( $\chi^{2}=25,08 p<$ $0,000)$ e a variável semanas de gestação passou de 6,0\%, em 1997, para 18,9\%, em $2005\left(\chi^{2}=\right.$ $65,343 p<0,001$ ) (Tabela 3).

$\mathrm{N}$ o que se refere às variáveis relacionadas ao momento do óbito, observou-se um decréscimo no número de municípios com preenchimento excelente, exceto a variável local de ocorrência, que apresentou um incremento, chegando a 2005 com todos os municípios atingindo esse escore. Identificou-se o pior desempenho no preenchimento das variáveis prestação de assistência mé dica ao agravo que levou ao óbito, passando de $33,3 \%$, em 1997, para 0,7\%, em $2005\left(\chi^{2}=141,927\right.$ $p<0,001)$ e realização de necropsia para confirmação da causa básica, passando de $26,0 \%$ dos municípios, em 1997, para 2,8\%, em 2005 ( $\chi^{2}=$ $54,838 p<0,001$ ) (Tabela 3).

\section{Discussão}

Apesar de ser o mais antigo dos sistemas de informação em saúde do país, o SIM ainda apresenta problemas que limitam sua utilização para a análise da mortalidade infantil e seus determinantes. Embora o Brasil possua um modelo padrão de declaração de óbito há mais de trinta anos, bem como um manual para instruir o preenchimento desse documento ${ }^{3}$, em Pernambuco não se verificou melhora no preenchimento de algumas variáveis referentes aos óbitos de me nores de um ano no período de 1997 a 2005.

0 monitoramento da mortalidade infantil depende da qualidade dos dados disponíveis no SI M , uma vez que os indicadores de saúderelacionadosà mortalidade são, em grandeparte, construídos com dados existentes nas declarações de óbito. Caso esses dados apresentem inconsistên- cias, podem prejudicar a confiabilidade dessas informações, gerando falsos diagnósticos de saúdeecomprometendo as medidas de intervenção. O M inistério da Saúde ${ }^{19}$ tem enfatizado a importância das estatísticas de mortalidade, por elas serem indispensáveis para a elaboração e análise dos principais indicadores de saúde.

Da declaração de óbito, constam importantes informações para o conhecimento dos principais determinantes da mortalidade infantil, como características socioeconômicas (ocupação e escolaridade materna), peso ao nascer, paridade, entre outros. Contudo, várias pesquisas vêm referindo deficiências no preenchimento dessas variáveis $5^{4,12,13}$, corroborando os achados do presente estudo.

Chama atenção o fato de que o elevado percentual de variáveis em branco/ignoradas pode mascarar os dados que aferem a qualidade da assistência prestada à gestante e ao recém-nascido, deixando deinformar corretamentereconhecidas variáveis preditoras do risco para o óbito, como peso ao nascer e idade materna. A pesar do peso ao nascer ter apresentado um acréscimo no número de municípios que atingiram o escore excelente, ainda são poucos os municípios de Pernambuco $(13,3 \%)$ que preencheram adequadamente esta variável em 2005. Pedrosa et al. ${ }^{13}$, em estudo sobre a qualidade dos dados dos óbitos neonatais precoces no município de Maceió (AL), nos anos de 2001 e2002, encontraram quea idadematerna foi a variável menos preenchida, che gando a $44,2 \%$ de omissão, e o peso ao nascer apresentou $15,2 \%$ de variáveis em branco/ignoradas. Observa-se que, apesar de ter sido estudado apenas os óbitos neonatais, que apresentam uma maior chance de preenchimento adequado da variável peso ao nascer, devido o óbito ter ocorrido muito próximo ao parto ${ }^{12,16}$, ainda é muito elevado o percentual depreenchimento em branco/ignorado dessas variáveis.

Por outro lado, o presente estudo constatou um aumento no número demunicípios com preenchimento excelentenas variáveis deidentificação da criança como sexo e idade. Em 2005, a quase totalidade dos municípios pernambucanosatingiu o escore excelente. Situação semel hante foi encontrada por H eckmann et al. ${ }^{10} \mathrm{em}$ estudo sobre o preenchimento da declaração de óbito no município deViamão (RS), no ano de 1987. Estes autores encontraram que, para as variáveis de identificação do falecido, o percentual de informação em branco/ignorado ficou abaixo de 10,0\%, devido à possibilidade do registro ser realizado diretamente com os familiares do falecido, Soares et al. ${ }^{4}$ encontraram um percentual de 
55,4\% de incompletude da variável sexo no município de Montes Claros (M G) no ano de 2005, demonstrando a diversificada realidade dos municípios brasileiros.

A distância temporal na realização dos referidos estudos pode ter influenciado os resultados encontrados. Apesar de se esperar uma meIhora no preenchimento das variáveis da declaração de óbito, vários estudos ${ }^{12,20}$ têm apontado para um aumento no percentual devariáveis com preenchimento em branco/ignorado. Estes achados refletem a pouca importância atribuída pelos médicos ao preenchimento adequado das declarações de óbito em sua totalidade, bem como o insuficiente investimento das instâncias gestoras do Sistema único de Saúde no que se refereà completitude desse documento, uma vez que as ações estão focadas, principalmente, na melhora da cobertura do SIM eno preenchimento da causa básica do óbito ${ }^{21}$.

Romero e Cunha ${ }^{12}$ identificaram problemas na instrução de preenchimento da declaração de óbito. Esses autores relatam que o manual de preenchimento traz instruções confusas em relação ao tratamento da informação ignorada, mesclando em uma mesma variável um código numérico atribuído à categoria "ignorado" com um caractere texto. A recomendação versa: "evitar deixar campos em branco, colocando o código correspondente a ignorado, ou um traço (-) quando não se conhecer a informação solicitada ou não se aplicar ao item correspondente" ${ }^{\prime \prime}$.

Além disso, consolidou-se entre os profissionais da área a idéia da existência de variáveis mais importantes, como o sexo, tipo de óbito e causa básica, em detrimento das demais $\varsigma^{4,10}$. Esta prática pode ter surgido a partir da classificação preconizada pelo M inistério da Saúde que separava as variáveis em indispensáveis, essenciais e secundárias²2. Estetipo de classificação "seleciona" as variáveis "mais importantes", trazendo a falsa impressão da pouca importância das demais variáveis, contribuindo com a cultura do não preenchimento.

Os maiores problemas identificados no presente estudo se referem exatamente às variáreis consideradas secundárias ${ }^{22}$ que, apesar de grande importância, não foram preenchidas de forma adequada. As condições sociodemográficas, tais como escolaridade eocupação materna, consideradas como fatores preditivos dos óbitos infantis, apresentaram um número reduzido de municípios com preenchimento excelente. Esse fato também foi constatado nas variáveis que tratam da paridade (número de filhos tidos) e aquelas referentes ao momento do óbito. Estes achados estão de acordo com diversos estudos que avaliaram a completitude da declaração de óbito ${ }^{4}, 10,12,13,16,19,20$.

Alguns autores apontam deficiências desde a geração atéa divulgação da informação pelo SIM , não se restringindo apenas ao precário preenchimento da declaração de óbito pelos médicos ${ }^{23}$. Prontuários com dados incompletos, registro inadequado das variáveis de identificação do falecido por técnicos administrativos e, até mesmo, problemas de digitação têm contribuído para a geração de dados incompletos ${ }^{8,9,11,19,20}$. Pedrosa et al. ${ }^{13}$ identificaram um elevado percentual de omissão na digitação de diversos campos da declaração de óbito, mesmo quando tinha sido marcada pelo médico, a exemplo da variável peso ao nascer, que apresentou $44,1 \%$ de omissão na digitação.

Essa falta de esclarecimento dos profissionais que manipulam a declaração de óbito leva à geração de dados incompletos, o que inviabiliza o pleno aproveitamento das informações. Essa prática obriga os profissionais da saúde pública a delinear estratégias de melhoria da qualidade da informação, como o resgate de dados no Sistema de Informações sobre N ascidos Vivos (SINASC), nos cartórios, nos hospitais, nos serviços deverificação de óbito, no instituto de medicinal legal, entre outros. Essas ações requerem um quantitativo de recursos humanos e materiais que muitas vezes não faz parte da realidade de muitos municípios do país.

Algumas estratégias necessitam ser adotadas no sentido de melhorar a completitude das variáveis da declaração de óbito, para que estas possam subsidiar análises da situação de saúde mais fidedignas. Como estratégias, destacam-se o fortalecimento da articulação entre asinstâncias gestoras do Sistema Único de Saúde euniversidades com o intuito de valorizar os currículos médicos e qualificar os profissionais atuantes quanto ao preenchimento correto, completo e fidedigno da declaração de óbito. Para tanto, é necessário a ênfase e o esclarecimento do papel social deste instrumento no que tange às estatísticas de saúde. Outra estratégia que pode ser utilizada no processo de qualificação processual dos médicos éa realização de estudos de mortalidade por unidade de saúde. Para tanto, o envolvimento dos núcleos de epidemiologia hospitalar nessa tar efa é uma alternativa a ser avaliada pelos gestores das unidades de saúde ${ }^{9,11}$.

Além dos médicos, é necessário instruir os funcionários administrativos e os digitadores 
sobre as normas, rotinas e fluxos do processamento da declaração de óbito², além de instituir uma rotina de atividades de supervisão, acompanhamento e controle de qualidade do SIM. Outra estratégia que vêm obtendo excelentes resultados, entre outros aspectos, na melhoria do preenchimento da declaração de óbito de menores de um ano éa atuação da Vigilância dos Ó bitos Infantis e dos Comitês de Prevenção do Ó bito Infantil ${ }^{24}$. Estes entes promovem a investigação e discussão sistemática dos óbitos ocorridos em determinada localidade, contribuindo para o aprimoramento da informação.

Este conjunto articulado de ações, se adotado de fato, pode levar em médio prazo a uma melhora nos registros do SIM , possibilitando o aproveitamento de todas as potencialidades desse sistema de informações em saúde.

\section{Colaboradores}

Costa JMB e Frias PG participaram de todas as etapas de construção deste artigo.

\section{Agradecimentos}

0 presente estudo foi financiado com recursos de um convênio firmado entre a Secretaria de Vigilância à Saúde SVS/M S e o Instituto M aterno Infantil de Pernambuco. 


\section{Referências}

1. Haraki CAC, Gotilieb SLD, Laurenti R. Confiabilidade do Sistema de informações sobre mortalidade em município do sul do estado de São Paulo. Rev. bras. Epidemiol. 2005; 8(1):19-24.

2. Brasil. Ministério da Saúde. Fundação Nacional de Saúde. M anual de Procedimentos do Sistema de Informações sobre M ortalidade. Brasília:M inistério da Saúde; 2001.

3. Brasil. Ministério da Saúde. Fundação Nacional de Saúde. M anual de Instrução para o preenchimento da declaração de óbito; 2001. Brasília: M inistério da Saúde; 2001.

4. Soares JAS, Horta FM B, Caldeira AP. Avaliação da qualidade das informações em declarações de óbitos infantis. Rev. Bras. Saude M ater. Infant. 2007; 7(3):289-295.

5. Scwarcwald CL, Leal MC, Andrade CLT, Souza Jr PRB. Estimação da mortalidade infantil no Brasil: 0 que dizem as informações sobre óbitos e nascimentos do Ministério da Saúde? Cad Saude Publica 2002; 18(6):1725-1736.

6. Paes NA. Avaliação da cobertura dos registros de óbitos dos Estados Brasileiros em 2000. Rev. Saude Publica 2005: 39(6):882-890.

7. Scwarcwald CL, Leal MC, Castilho EA, Andrade CLT. Mortalidade infantil no Brasil: Belíndia ou Bulgária? Cad Saude Publica 1997; 13(3):503-516.

8. Frias PG, Vidal AS, Pereira PMH, Lira PIC, Vanderlei LC. Avaliação da notificação de óbitos infantis ao SIM : um estudo de caso. Rev. Bras. Saude Mater. Infant. 2005; 5 (Supl. 1):S43-s52.

9. Vanderlei LC, Arruda, BKG, Frias PG; Arruda S. Avaliação da Confiabilidade da causa básica de óbito em unidade terciária de atenção à saúde materno infantil. Inf Epidemiol SUS 2002; 11(1):15- 23.

10. Heckmann IC, Canani LH, Sant'anna UL, Bordin R. Análise do preenchimento de declarações de óbitos em localidade do estado do Rio Grande do Sul (Brasil), 1987. Rev. Saude Publica 1989; 23(4):292-297.

11. Vanderlei LC, Arruda BKG, Frias PG, Arruda S. Avaliação da Qualidade do preenchimento das DO em unidade terciária de atenção à saúde materno infantil. Inf Epidemiol SUS 2002; 11(1):7-14.

12. Romero $D E$, Cunha $C B$. Avaliação da qualidade das variáveis sócio-econômicas e demográficas dos óbitos de crianças menores de um ano registrados no Sistema de Informações sobre mortalidade do Brasil (1996/2001). Cad Saude Publica 2006; 22(3):673-684.

13. Pedrosa LDCO, Sarinho SW, Ximenes RAA, Ordonha MR. Qualidade dos dados sobre óbitos neonatais precoces. Rev Assoc M ed Bras 2007; 53(5):389394.

14. Andrade CLT, Szwarcwald CL. Desigualdades sócio-espaciais da adequação das informações de nascimentos e óbitos do Ministério da Saúde, Brasil, 2000-2002. Cad SaudePublica 2007; 23(5):1207-1216.
15. Organização Pan-Americana da Saúde. Rede Interagencial de Informação para Saúde. Indicadores Básicos de Saúde no Brasil: conceitos e aplicações. Brasília: Organização Pan-Americana da Saúde; 2002. p. 315-316.

16. Almeida M F, Alencar GP, Novaes HMD, Ortiz LP. Sistemas de informação e mortalidade perinatal: conceitos e condições de uso em estudos epidemiológicos. Rev. bras. Epidemiol. 2006; 9(1):56-68.

17. Brasil. M inistério da Saúde. Datasus. [site da Internet]. [acessado 2007 nov 11]. Disponível em: http:/ /msbbs.datasus.gov.br/public/default.htm

18. Brasil. Assembléia Legislativa do Estado de Pernambuco. Lei Complementar no 1 , de 12 de julho de 1990. Dispõe sobre requisitos para criação de M unicípios, e dá outras providencias. [site da Internet] [acessado 2008 mar 3]. Disponível em: http:// legis.alepe.pe.gov.br/legis_superior_norma.aspx? $\mathrm{nl}=\mathrm{LC} 001$

19. Brasil. Ministério da Saúde. Fundação Nacional de Saúde. M ortalidade Brasil 1994. Brasília: Ministério da Saúde; 1997.

20. Barros M DA, Ximenes R, Lima M LC. Preenchimento das variáveis nas declarações de óbitos por causas externas de crianças e adolescentes no Recife, de 1979 a 1995. Cad Saude Publica 2001; 17(1):71-78.

21. Brasil. M inistério da Saúde. Sistemas de Informações sobre mortalidade e nascidos vivos para os profissionais do programa de Saúde da família. 2ª ed. Brasília: Ministério da Saúde; 2004.

22. Brasil. Ministério da Saúde. Fundação Nacional de Saúde. Manual de instrução para o preenchimento da declaração de óbito; 1997. Braślia: M inistério da Saúde; 1997.

23. Frias PG, Pereira PM H, Andrade CLT, Scwarcwald $\mathrm{CL}$. Sistema de Informações sobre M ortalidade: estudo de caso em municípios com precariedade de dados. Cad Saude Publica 2008; 24 (10):2257-2266.

24. Mansano NH, Mazza VA, Soares VM N, Araldi MAR, Cabral VLM . Comitês de prevenção da M ortalidade Infantil do Paraná, Brasil: implantação e operacionalização. Cad Saude Publica 2004; 20(1):329-332.

Artigo apresentado em 28/08/2008

A provado em 20/06/2009

Versão final apresentada em 20/07/2009 